\title{
Diversos pero concentrados: percepciones de comunicadores sobre el pluralismo de los medios digitales en Chile ${ }^{1}$
}

Diverse but concentrated: perceptions of communicators on the pluralism of digital media in Chile

\section{Nicolás del Valle}

International Institute for Philosophy and Social Studies, Santiago, Chile

ndelvalleaiipss.com

https://orcid.org/0000-0002-7083-7928

\section{Fernando Carreño}

International Institute for Philosophy and Social Studies, Santiago, Chile

fcarrenodiipss.com

https://orcid.org/0000-0002-1067-491X

\section{Resumen}

Este artículo analiza las percepciones de los comunicadores de los medios digitales de acuerdo a sus discursos sobre la situación del pluralismo informativo en Chile. Las opiniones de los comunicadores se recopilaron mediante 19 entrevistas que revelan las condiciones subjetivas de profesionales de diferentes medios digitales del país. Los resultados obtenidos indican que los periodistas entienden al pluralismo informativo como una diversidad mediática, considerando a su propio medio de comunicación como pluralista internamente, aun cuando se percibe que el sistema de medios en su conjunto ve limitado su pluralismo por la comercialización de los medios y el poder del dinero sobre las líneas editoriales.

Palabras clave: periodismo, pluralismo informativo, medios digitales, Chile.

\begin{abstract}
This article analyzes the perceptions of communicators of digital media according to their discourses on the situation of media pluralism in Chile. The opinions of the communicators were collected through 19 interviews that reveal the subjective conditions of different digital media in the country. The obtained results indicate that journalists understand media pluralism as a media diversity. Also, they consider their own media as pluralistic internally, even when it is perceived that the pluralism of the media system as a whole is limited by the commercialization of the media and the power of money over editorial lines.
\end{abstract}

Keywords: journalism, Media Pluralism, Digital Media, Chile. 


\section{Introducción}

Este artículo analiza las percepciones de los comunicadores de los medios digitales en sus discursos sobre la situación del pluralismo informativo en Chile. El punto de vista de los sujetos detrás de los medios de comunicación es una dimensión central en las investigaciones sobre el pluralismo informativo, aun cuando los estudios sobre el caso chileno han estado centrados principalmente en el consumo de información, las audiencias y las políticas públicas (Arriagada, Correa, Scherman \& Abarzúa, 2015; Correa, Scherman \& Arriagada, 2016; Zárate, 2016). Algunos estudios cualitativos han destacado la importancia de las condiciones subjetivas para la comprensión de las percepciones de los periodistas respecto de la situación del pluralismo, así como sobre los fenómenos de la producción de contenidos, las prácticas periodísticas y las dinámicas internas de los medios (Sapiezynska, Lagos \& Cabalin, 2013). En esta última dirección, este artículo espera caracterizar las percepciones de los comunicadores dedicados a la edición, gestión y dirección de los medios digitales escritos en torno al pluralismo informativo, identificando las regularidades discursivas por medio de códigos emergentes en entrevistas.

¿Cuáles son las percepciones de los comunicadores de los medios digitales escritos respecto de la situación del pluralismo informativo? Según la opinión de los comunicadores ¿los medios digitales se desenvuelven en un sistema pluralista? En suma, ¿cuáles son los discursos de quienes dirigen, editan y gestionan los medios respecto del pluralismo informativo en Chile? La evidencia sostiene que los comunicadores conciben al pluralismo informativo en tanto que diversidad en los medios, pero se ve restringido empíricamente por la concentración económica y del poder comunicativo. En otras palabras, los discursos afirman que el pluralismo contribuye al ejercicio del periodismo y la democracia, pero las condiciones actuales del sistema informativo representan una amenaza real a su realización. Esta percepción es reforzada con las tendencia de precarización laboral de los periodistas con la fusión de salas de redacción para la producción de contenidos digitales y la creciente polifuncionalidad de los comunicadores detrás de los medios digitales.
Para responder las preguntas planteadas, se elabora un cuestionario semiestructurado que es aplicado en entrevistas en profundidad con el fin de registrar las perspectivas de comunicadores de diversos medios que transmiten información escrita a través de plataformas digitales, incluyendo tanto los nuevos medios autogestionados como los medios de comunicación tradicionales dependientes de sus versiones impresas. Sin despreciar los estudios tradicionales dedicados al pluralismo informativo desde mediciones agregadas y cuantitativas, este artículo se enfoca en la dimensión reflexiva de los agentes dedicados a comunicar e informar; es decir, consiste en el análisis e interpretación de sus discursos para caracterizar el pluralismo en los medios en sus diferentes componentes. Con ello, se espera colaborar con una agenda de investigación sobre las percepciones de los periodistas respecto del pluralismo informativo en Chile. Las preguntas del cuestionario abarcan tópicos como la situación del pluralismo, la ética periodística, línea editorial del medio, el rol de los medios digitales en la sociedad, más otros aspectos del sistema informativo nacional.

El itinerario de este artículo consiste en introducir la discusión política y académica sobre el pluralismo informativo en orden de definir los componentes del concepto y definir el marco teórico en el cual se inserta esta investigación. En un segundo momento, se abordará el conjunto de procedimientos metodológicos de las entrevistas semiestructuradas, desde el periodo de tiempo en el cual se aplicaron hasta los medios que fueron cubiertos. Luego, se interpretan los discursos de los editores, directores y gestores de contenidos de los medios, teniendo especial atención en las regularidades del discurso de acuerdo con los componentes del pluralismo informativo y las menciones más frecuentes en las entrevistas (frecuencia de códigos). Finalmente, se proponen algunas observaciones sobre las opiniones y valoraciones del pluralismo informativo.

\section{Pluralismo informativo, periodismo y medios digitales}

De acuerdo al uso general del concepto de pluralismo, este suele ser confundido con una pluralidad de puntos de vista, intereses, ideas y reali- 
dades que son representadas en las agendas, los contenidos y las líneas editoriales de los medios de comunicación. Contraria a esta concepción del pluralismo que suele reducirlo a mera heterogeneidad al interior de los sistemas informativos, la propia teoría del pluralismo democrático ha sostenido que éste es irreductible a la diversidad (Dahl, 1991; Rawls, 1997, 1993; Sartori, 2001), planteando la pregunta por la relación entre diversidad y pluralismo.

El pluralismo supone una sociedad diversa internamente dado la diferenciación estructural de los procesos de modernización social. El pluralismo informativo emerge en sociedades complejas en las cuales los individuos, las organizaciones y los campos sociales se desarrollan con relativa autonomía. Así, el pluralismo no es solo una situación social (multiplicidad, heterogeneidad o pluralidad de perspectivas), sino un conjunto de principios reguladores que rigen las dinámicas entre las diferencias. Una sociedad de castas o estamentos puede considerarse una sociedad heterogénea pero no por ello pluralista, del mismo modo que un sistema informativo diverso en términos de la oferta informativa tampoco lo es necesariamente.

El carácter normativo del pluralismo es destacado por Kari Karppinen (2013) quien lo distingue como “un ismo, [que] refiere más explícitamente a un valor de orientación que considera la multiplicidad y la diversidad una virtud en ideas e instituciones" (Karppinen, 2013, p. 4). Siguiendo lo anterior, la diversidad es la existencia y reconocimiento de dicha heterogeneidad. Para nuestra investigación, la diversidad es entendida como la expresión de la pluralidad de intereses, ideas, opiniones y realidades en los medios que forman la opinión de los ciudadanos.

Sin profundizar en las distintas implicaciones conceptuales y empíricas, las cuales han sido desarrolladas en otros momentos (Del Valle, 2016a, 2016b), cabe señalar que el pluralismo informativo también comprende a la libertad en los medios, esto es, las libertades de expresión y de prensa, así como la autonomía de los medios de comunicación frente a los poderes económicos o políticos. Teóricamente esto se debe a que la diversidad en la esfera pública es el resultado de un proceso creciente de individuación de los ciudadanos, habilitándolos a participar en el debate público y ejercer sus libertades civiles de expresión y asociación (Habermas, 1998; Honneth, 2014, pp. 255-304). La existencia de las libertades, ya sean civiles o políticas, es la garantía para la manifestación de la diversidad como para proteger dichas expresiones. Dicho lo anterior, es razonable sostener que la libertad en los medios es un componente del pluralismo en tanto que garantías para la difusión organizada de información, ideas y opiniones sin restricciones externas arbitrarias.

Ahora bien, la garantía del libre acceso a la esfera pública no significa un resultado pluralista a la hora de evaluar los sistemas informativos. Esto requiere de un ejercicio efectivo de dichas libertades a través de la participación en los medios (Boussaguet, 2016). Este enfoque comprende a la libertad como una potencia de diversidad en potencia pero que requiere que los ciudadanos emprendan nuevos medios y formen parte de la producción informativa de los ya existentes. La participación voluntaria de las audiencias en la producción de los contenidos en una relación de simultaneidad digital, es otro componente de nuestro concepto (Del Valle, 2016a, p. 13).

Para una realización plena del pluralismo informativo tienen que atenderse las condiciones estructurales, ya sea que permitan el desarrollo mediático como que protejan la manifestación de diversidad, el ejercicio de la libertad y la participación en los medios. Esta es la postura sostenida por Nicholas Garnham $(1992,1999)$ quien tensiona al pluralismo respecto de la distribución del poder. Pues a pesar de las garantías para la libertad de expresión, la manifestación de la diversidad y el ejercicio efectivo de la participación, las asimetrías del poder comunicativo son clave para definir la incidencia en las agendas políticas, así como afectar la capacidad de los actores para participar del debate público.

Por ejemplo, la concentración de la propiedad y el control disminuye la competencia y el pluralismo desde un nivel global (Stühmeier, 2016), pasando por una óptica desde América Latina (Becerra \& Mastrini, 2017) y Chile (Breull, 2015), además considerando en esta misma línea lo presentado en el trabajo de Sergio Godoy y María Elena Gronemeyer sobre los medios digitales en Chile y la relevancia del financiamiento privado (2012). Correlacionando la discusión de la literatura secundaria y los 
hallazgos, esta tensión también se constata en las entrevistas donde se señala que las fuentes de financiamiento afectan directamente las capacidades de comunicar y la permanencia y composición de los equipos editoriales, incidiendo incluso en la percepciones de los periodistas (Hatcher \& CurrinPercival, 2016; Domingues-da-Silva, 2015).

Por poder comunicativo se entiende "la capacidad relacional que permite a un actor social influir de forma asimétrica en las decisiones de los otros actores sociales en formas que favorecen la voluntad, intereses y valores del actor empoderado" (Castells, 2009, p. 10). Además, el mismo Castells lo identifica como base fundamental de la sociedad (2015). 0 en palabras de Karppinen, dicho poder de comunicar es "la capacidad de un actor social de movilizar significativamente la comunicación de acuerdo a exceder dicha influencia" (Karppinen, 2013, p. 611. Entonces, el pluralismo informativo sería afín a una política distributiva del poder en las comunicaciones, es decir, iniciativas regulatorias que promuevan una participación equitativa en el debate público, minimizando la influencia de los propietarios y controladores de los medios para dejar espacio al libre ejercicio del periodismo. Un sistema informativo pluralista, por lo tanto, se tensiona con la concentración económica de la industria de medios.

Con esta visión sinóptica de nuestro concepto a partir de sus componentes (diversidad, libertad, participación y distribución), cabe preguntarse cuáles son los alcances empíricos. Aún cuando el objetivo de este artículo no es aplicar dicho concepto para medir la realidad, sí es relevante definirlo como el "grado de comunicación libre y equitativa de diversos intereses, ideas y realidades a través de una transmisión participativa de la información" (Del Valle, 2016a, p. 14), pues esto permite contar con una definición que incluye todos los componentes proveyendo un rendimiento analítico.

\section{Pluralismo en los medios digitales}

Ahora bien, ¿cómo se relaciona el pluralismo informativo y los medios digitales escritos? La emergencia de nuevos medios digitales ha planteado un conjunto de desafíos a la regulación de las comuni- caciones y la información (Duarte, Huertas, Rosa \& Caffarena, 2015). Según Castells (2001), la era de la información implica un nuevo tipo de comunicación caracterizada por la simultaneidad. En el caso de los medios digitales, éstos se diferencian de otros medios como la televisión, la radio y los periódicos impresos debido a la simultaneidad. No sólo la comunicación entre los comunicadores y las audiencias se ha vuelto inmediata gracias a las nuevas plataformas digitales, tales como los sitios web o las redes sociales (Campos-Freire, Rúas-Araújo, López-García \& Martínez-Fernández, 2016; VarasAlarcón \& González-Arias, 2016), sino además el ejercicio de las prácticas informativas ha incluido esta simultaneidad entre los hechos y contenidos noticiosos (Klinenberg, 2005).

Mientras que se ha sostenido que el uso del internet en los medios de comunicación significa una mayor participación de la ciudadanía en las agendas noticiosas, garantizando la libertad de expresión y de prensa (Clemens, 2010; Czepek, 2009; Kahne \& Middaugh, 2012; McNeal, Mossberger \& Tolbert, 2007; Nielsen, 1984); al mismo tiempo, significa un avance de las empresas desde los formatos tradicionales a los nuevos medios online implicando una mayor concentración (Almiron, 2006) y una disminución de la diversidad de temas, posturas y formatos (Grazian, 2005).

De manera que la digitalización de las comunicaciones amplía las oportunidades para expresar opiniones públicamente, así como diversifica los canales de información y de participación de las audiencias. La participación de los ciudadanos en los medios de comunicación es un aspecto ineludible y además deseable en el mundo actual. Medios que incluyen a las audiencias en la producción de los contenidos aseguran una diversidad de fuentes, temas y posturas en las agendas noticiosas, junto con proveer canales de información más independientes del poder político y económico, insertando nuevas modalidades de presentación de la realidad que traspasan una disposición discursiva que pueda inhibir y clausurar el propio pluralismo informativo.

Actualmente, es posible responder, compartir y rectificar los contenidos de los medios, al mismo tiempo que publicar una noticia sobre un hecho ocurrido por "periodistas ciudadanos". En este contexto, las audiencias participan en el proceso editorial que selecciona las noticias de acuer- 
do a sus preferencias, lectorías y comentarios, transformando a los públicos en comunicadores al momento de la cobertura de hechos noticiosos y la producción de contenidos (Coddington, 2012; Welbers, Van Atteveldt, Kleinnijenhuis, Ruigrok \& Schaper, 2016).

Estos cambios que trajeron consigo los nuevos medios digitales, implican transformaciones en la dinámicas de diversos tipos de medios. Los nuevos canales de televisión y radio online, los podcast, los blogs y diarios digitales, pueden ser incluidos en la emergencia de nuevos medios de comunicación (Domingo \& Heinonen, 2008). En este último sentido, sería correcto catalogar a todas estas nuevas modalidades como medios digitales, independiente del tipo de información transmitida lescrita, audio y visual).

En este artículo, los hallazgos de investigación se centran en una familia particular de los medios digitales: los medios digitales escritos (MDE). Están centrados en la transmisión de información escrita, a diferencia de otros medios digitales como la televisión y la radio online (Del Valle, 2016a; Domingo \& Heinonen, 2008). Sin embargo, cabe distinguir entre los medios digitales que transmiten información escrita a través de un proceso editorialperiodístico y aquellos que no lo hacen. En efecto, el surgimiento de nuevos canales de información incluye el auge de blogs y diarios digitales entre los cuales no hay un proceso periodístico de acuerdo a los estándares de una línea editorial (Wall, 2005), la constatación de los hechos, el contraste de puntos de vistas y las fuentes informativas, e información con pretensiones de imparcialidad (Dreyfus, Lederman, Bosua \& Milton, 2011). Esta realidad es cada vez más pronunciada en los estudios sobre el auge de noticias falsas y sus impactos en la opinión pública (Sánchez-Gonzales \& Méndez-Muros, 2015). Por tales motivos, un MDE se concibe como un canal online que transmite información escrita a través de un proceso editorial en el cual la comunicación entre las audiencias y los comunicadores es simultánea (Del Valle, 2016a, p 9). Como se indica en el apartado metodológico, con este criterio es posible delimitar el universo, dejando fuera a sitios web que, aunque transmiten información escrita, lo hacen sin procesos editoriales.

Asimismo, si hablamos de los MDE, el concepto tradicional de periodismo debe ser ampliado para la comprensión de este nuevo fenómeno. Con la aparición de los nuevos medios, paulatinamente el periodismo se ha convertido en una actividad que puede abarcar un conjunto de prácticas que exceden la profesión de los periodistas. En la actualidad, la emergencia de nuevos medios gestionados por organizaciones de comunicadores no profesionales plantea otros conceptos sobre el ejercicio del periodismo. El "nuevo periodismo" (Fenton, 2010), el "periodismo ciudadano" (Wall, 2015) y el "periodismo digital" (Scott, 2005) se repiten a lo largo de la historia reciente de los medios digitales, los cuales se caracterizan por la inclusión de nuevos agentes y prácticas en la producción de la información periodística (Domingo \& Heinonen, 2008; Hansen, 2013; Lloyd, 2015). De este modo, la actividad de comunicar a través de los medios se desplaza hacia un conjunto de prácticas que no se reducen al periodismo tradicional y restringido.

Por lo tanto, periodismo comprendería una paradoja en la era digital que puede resumirse en dos sentidos distintos (Hansen, 2013). Un primer concepto restringido y formal del periodismo lo concibe como el conjunto de prácticas realizadas por los profesionales de las comunicaciones. En el caso chileno, según la legislación vigente, estos coinciden con las personas que ostentan el título profesional de periodista (Martinez-Arias, 2015). Por otro lado, se encuentra un concepto amplio del periodismo que no se adscribe al conjunto de prácticas determinadas por un estatus profesional o adscripción gremial (Fortunati \& Sarrica, 2011). Por el contrario, por periodismo se entiende toda práctica que tiene como objetivo informar pública e imparcialmente a través de los medios (Wall, 2015), democratizando los medios de comunicación y afectando sus modelos de negocios (Zeng, Dennstedt \& Koller, 2016).

\section{Metodología}

El uso del concepto de MDE permite circunscribir el estudio con criterios metodológicos que delimitan el alcance de la investigación y definen las unidades de análisis de manera concreta. Los criterios que sirvieron para la selección de los medios correspondían a canales de información definidos (1), que transmiten principalmente contenido escrito (2) y que producen contenido nativo del medio (3) a través de relaciones de simultaneidad con sus 
públicos (4). Con estos criterios se asegura que la muestra se caracteriza por medios con un trabajo editorial en el centro de la proliferación de proyectos periodísticos informales en la era digital.

Con los criterios anteriores se define un campo que debe acortarse debido a la gran cantidad de MDE que encajan con la categoría. Atendiendo al pluralismo informativo y a las características dinámicas de la esfera digital, también se incluyen a medios con una antigüedad de al menos dos años (5) y con contenidos que tengan una periodicidad diaria o semanal (6), así como que cuenten con un equipo editorial (7) y publiquen contenido político (8). Con ello, se espera que los medios participantes en esta investigación sean canales de información estables en el tiempo y que contribuyan al debate político desde el periodismo.

Una segunda consideración metodológica atiende a la delimitación territorial a nivel nacional. Dado la centralidad de lo digital en la investigación, se consideró a las tres regiones con mayor penetración de internet, a saber, las regiones V, VIII y Metropolitana, en el entendido que el acceso del internet es una de las condiciones de la proliferación medios digitales. Como es de esperar, dichas regiones también coinciden con los territorios con mayor densidad demográfica (IDC, 2009, 2012). De estas tres regiones y siguiendo los criterios antes mencionados, la muestra total está compuesta por un total de 66 medios.

De manera no probabilística se establece una muestra compuesta por MDE de las regiones recién mencionadas y que cumplen con los criterios ya establecidos. En este marco se aplicaron entrevistas semi-estructuradas a miembros de 19 medios que participaron en el proceso editorial de los contenidos políticos. La cantidad de entrevistas estuvo determinada tanto por el nivel de respuesta como por la aspiración de representar la pluralidad de los MDE y así contar con material relevante para la comprensión del fenómeno.

La entrevista consta de un cuestionario semiestructurado de 30 preguntas abiertas que fue aplicado el año 2014 a partir de los elementos del pluralismo informativo ya descritos. Así, por cada componente del concepto, se ordenan una serie de preguntas orientadas a recabar información cualitativa sobre las opiniones, apreciaciones y valoraciones de los periodistas sobre el pluralismo en el sistema informativo nacional. Las preguntas abarcan tópicos como la situación del pluralismo, la ética periodística, las líneas editoriales, entre otros aspectos.

El trabajo de terreno supuso una estrategia de acercamiento a los diferentes medios que participaron en el estudio. La aplicación de los cuestionarios se detuvo luego de la saturación discursiva de las entrevistas y conforme se compuso una muestra equilibrada en cuanto a los tipos de MDE.

Se realizaron entrevistas a comunicadores de MDE dependientes de grandes conglomerados e independientes, medios comunitarios y de cobertura nacional, de periodismo informativo y de investigación. Cada una de ellas fue realizada de manera presencial y tuvieron una duración promedio aproximado de 25 minutos. Las y los participantes de estas entrevistas fueron principalmente Directores/as, Editores/as y Encargados de secciones. Los medios incluídos se presentan en la Tabla 1.

Tabla 1. Medios digitales estudiados

\begin{tabular}{|c|c|c|}
\hline Medio & Región & Sitio web \\
\hline Acuerdos & Metropolitana & acuerdos.cl \\
\hline Chile B & Metropolitana & chileb.cl \\
\hline CIPER & Metropolitana & ciperchile.cl \\
\hline Diario Financiero & Metropolitana & df.cl \\
\hline Diario U. de Chile & Metropolitana & radio.uchile.cl \\
\hline Dichato al Día & Biobío & dichatoaldia.cl \\
\hline El Concecuente & Biobío & elconcecuente.cl \\
\hline El Dínamo & Metropolitana & eldinamo.cl \\
\hline El Martutino & Valparaíso & elmartutino.cl \\
\hline El Periodista & Metropolitana & elperiodista.cl \\
\hline El Quinto Poder & Metropolitana & elquintopoder.cl \\
\hline El Resumen & Biobío & elresumen.cl \\
\hline La Nación & Metropolitana & lanacion.cl \\
\hline La Pulenta & Metropolitana & lapulenta.cl \\
\hline La Tercera online & Metropolitana & latercera.com \\
\hline Publimetro & Metropolitana & publimetro.cl \\
\hline Putaendo Informa & Valparaíso & putaendoinforma.com \\
\hline Soy Chile & Metropolitana & soychile.cl \\
\hline The Clinic & Metropolitana & theclinic.cl \\
\hline
\end{tabular}

Fuente: Elaboración propia 
Para la selección de los entrevistados se da prioridad a los perfiles que mantienen un grado importante de participación en la producción de contenidos. En cuanto al análisis del material, se identifican las regularidades en los discursos de los comunicadores sobre el pluralismo informativo. Posteriormente se procede a registrar las menciones con mayor frecuencia ( $f$ ) a partir de su codificación. La codificación emerge desde los discursos y no fue resultado de una búsqueda predeterminada, demostrando la coherencia del concepto pero también cómo sus componentes se relacionan entre sí. Los principales ejes discursivos y los temas mayormente abordados son discutidas en el análisis del material cualitativo.

\section{Resultados}

\subsection{Pluralismo como diversidad}

Casi la totalidad de las entrevistas (18) dan cuenta que el pluralismo informativo es entendido mayoritariamente como una diversidad de perspectivas representadas en los medios. En esta referencia se constata la idea de dar cabida a distintas "miradas", "voces", "posturas", "opiniones" y "realidades". Uno de los entrevistados, que es director y editor de un medio de la Región Metropolitana (medio $\mathrm{n}^{011}$ ), menciona esto con bastante claridad: "el pluralismo es darle espacio a todas las opiniones".

La concepción del pluralismo como diversidad se estabiliza en el discurso de los comunicadores, expresándose en la relevancia que se le otorga al componente de "diversidad", donde el código identificado con mayor frecuencia tiene relación con: ¿Qué entiende el medio por pluralismo? ( $f=37$ ), siendo este aspecto el principal dentro de la familia de códigos identificados con el componente diversidad $(f=121)$. En este mismo sentido, también se observa que el valor de la diversidad acantona en aspectos tales como el posicionamiento de temas en la agenda política, y que vayan más allá de lo estrictamente nacional, valorando las realidades locales. El director de prensa de un medio de la Región de Valparaíso (medio $n^{\circ} 8$ ) menciona lo siguiente: "la línea editorial del diario reconoce las diferentes realidades que hay en nuestro entorno donde estamos ubicados qué es la comuna de Putaendo". Apuntando a un aspecto similar desde el rol del medio como agente de visibilidad de problemáticas locales, distintas a la concentración de los grandes conglomerados, un entrevistado de la Región del Biobío (medio n ${ }^{0} 14$ ) menciona lo siguiente: "Este tema es sumamente importante para nosotros en contraste para los medios que son parte del duopolio en los cuales este tipo de temas no existe". Estos aspectos del discurso de los comunicadores confirman que el pluralismo entendido como diversidad contempla dos aspectos relevantes: diversidad de ideas y diversidad en términos de exposición de realidades locales.

Junto a lo anterior, es posible identificar que el pluralismo informativo como diversidad también se comprende según otras dimensiones que forman parte del concepto, a saber, la concentración de la propiedad de los medios ( $f=37$ ), que acá se considera parte de la dimensión denominada "distribución del poder comunicativo". Específicamente sobre la relevancia y efectos de la concentración de los medios, destaca la palabra de la editora de un medio de la Región Metropolitana (medio n017), quien condensa el argumento en torno a la concentración de los medios. Ella señala:

Muy nocivo [refiriéndose a la concentración], yo creo que es un efecto en cadena bien amplio. La manipulación de los medios de comunicación manipula finalmente las decisiones de la gente en su vida diaria en relación al consumo, a las elecciones políticas, sobre sus relaciones familiares, su mirada sobre asuntos, su relación con el medio ambiente porque finalmente la opinión se forma a través de los medios de comunicación y las relaciones con la familia. Cuando tú tienes manipulados los medios de comunicación o no permites que la gente acceda a todas las miradas sobre un tema, ese tipo de decisiones tan particulares desde dónde compro algo hasta por quién voto está absolutamente vinculado a la falta de diversidad y pluralismo.

Se observa que las relaciones entre los componentes permiten iluminar la categoría central de este estudio desde el punto de vista de los comunicadores. Por tanto, si bien es bastante claro que el pluralismo se entiende en gran medida como diversidad, no sólo se asocia con la voluntad y disposición por poner en lo público diferentes voces, también tiene estrecha relación con la valoración entregada 
a aspectos propios como la concentración, tanto a nivel geográfico y económico, y su relevancia para el sistema y las audiencias. En el estudio realizado por Arriagada et al. (2015) podemos constatar que las audiencias de regiones distintas a la Región Metropolitana valoran en buena medida la producción de información desde los medios locales (o regionales), pues consideran que aquellos no tienen cabida en medios de alcance nacional, marcando un alto grado de concentración (p. 72). Este aspecto es importante al momento de identificar que en los discursos también se menciona la concentración como un aspecto que se opone a la diversidad, aún cuando el pluralismo como diversidad se considera un valor fundamental para el sistema de medios.

\subsection{Límites del pluralismo informativo: concentración económica y del poder comunicativo}

A un nivel general, los entrevistados mencionan que la concentración de medios ocurrida en las últimas décadas ha generado efectos contraproducentes para el pluralismo informativo. En relación a este punto, la dimensión de la concentración económica y del poder comunicativo es la "distribución". En ella identificamos que las familias de códigos corresponden a una frecuencia discursiva menor a la de diversidad ( $f=95$ ), sin embargo, en lo que respecta a la "concentración", la frecuencia del código es la misma sobre lo que el medio entiende por pluralismo ( $f=37$ ). Por tanto, diversidad y concentración se configuran en los aspectos más relevantes para el análisis.

En este aspecto, el director de un medio de la Región Metropolitana (medio $\mathrm{n}^{0} 2$ ) indica:

La concentración le ha hecho mal al sistema de medios, a la política, y a la vida ciudadana de este país. Por eso yo he planteado que una de las soluciones es que el gobierno distribuya mejor sus platas.

Además, menciona en otro punto que los MDE “tienen que aprovechar el espacio que poseen, espacio que se han ido ganando en pro de ir a desarrollar más la discusión política en este país concentrada en los aspectos que presenta El Mercurio o La Tercera".
Desde una perspectiva de la diversidad, uno de los entrevistados menciona que una ausencia de pluralismo se expresa en una dependencia comercial, apuntando a los modos de financiamiento. Quien ejerce como editor de uno de los medios (medio $n^{03}$ ) considera que "esa dependencia [comercial] hace imposible una expresión verdadera de pluralismo en cuanto medio de comunicación". Este aspecto nos permite ver que a pesar de reconocer al pluralismo como diversidad, la consideración de su puesta en acto es problemática cuando se ponen en juego aspectos propios al financiamiento.

Otro aspecto a destacar son los riesgos que constituyen una amenaza a la realización del pluralismo. En este sentido, dos códigos discursivos que componen (junto a otros) la dimensión de distribución, fuentes de financiamiento $(\mathrm{f}=20)$ y transparencia ( $f=8$ ), también forman parte del discurso. En relación a eso, es menester destacar lo expuesto por el editor de un MDE (medio $\mathrm{n}^{0}{ }^{19}$ ) dedicado a la investigación periodística:

\footnotetext{
Es importante indagar en aspectos concretos del oficio, cómo se ejerce y de qué manera se relaciona con aspectos dominantes. Por ejemplo: ¿avisadores o abusadores? ¿Cómo influyen en la práctica las empresas que pagan por avisaje en los contenidos y orientaciones de la información que publica el medio? En concreto, el periodista que dirige o edita un medio ¿recibe regalos, premios, invitaciones? ¿Influye eso en la producción de sus contenidos? Hay que cuestionar esos aspectos y contrastarlos con los contenidos escritos.
}

Concentración, fuentes de financiamiento y transparencia muestran el contorno del discurso de los comunicadores en relación a los límites del pluralismo actualmente. De ese modo, el diagnóstico por parte de los comunicadores de los MDE ubica al "oligopolio en términos del control de los grandes medios" (medio $\mathrm{n}^{0} 14$ ) como uno de los mayores riesgos al pluralismo informativo en el país. Esta relación opuesta entre pluralismo informativo y concentración de la propiedad y el control de los medios se constata en la totalidad de las entrevistas.

De todas formas, casi todos los comunicadores (18) afirmaron que sus medios eran pluralistas, exponiendo que a pesar de las restricciones y ausencias de un pluralismo en el sistema, internamente los 
MDE no carecen de pluralismo. Esto último, puede ser relacionarse con la potencialidad del Internet y las plataformas digitales para un sistema pluralista. De acuerdo a algunos entrevistados, "ha habido más pluralismo gracias a la tecnología y a las redes sociales" (medio ${ }^{0}{ }^{0}$ ), reflejado en que "hoy día enfrentamos lo que es el surgimiento de diferentes medios alternativos más informales lo que nos ha facilitado la existencia de una variedad de medios" (medio $\mathrm{n}^{\circ} 8$ ). Sin embargo, a pesar que "cada vez hay mayor pluralismo por el manejo que hay de las redes sociales [...] si bien hemos avanzado en Chile, no existe pluralismo como se expresa en otras sociedades" (medio n ${ }^{011}$ ). Esto último, nos permite dar cuenta de la función de las redes sociales para un mayor nivel de participación, aún cuando el discurso que el sistema de medios adolece de pluralismo es abrumador en la muestra.

\section{Conclusiones}

El pluralismo informativo ha devenido un valor fundamental para el ejercicio del periodismo y una situación deseable para las sociedades democráticas. Esto es corroborado tanto en la bibliografía especializada como en las políticas de medios en perspectiva comparada, pero también desde la opinión de los comunicadores detrás de los medios digitales. Para los entrevistados el pluralismo informativo corresponde a una diversidad de voces al interior de los medios de comunicación y en el sistema medial en su conjunto, reduciendo el pluralismo a una cuestión de diversidad de contenidos, temas y líneas editoriales. Este es uno de los principales resultados luego del análisis de los datos levantados.

En este contexto, la percepción de los comunicadores es bastante clara en cuanto a la situación del país. Según los hallazgos mencionados, el pluralismo no sólo es una situación deseable que debería guiar las acciones públicas en el sector de las comunicaciones y la información, sino que se ve amenazado en Chile por factores estructurales como los modelos de financiamiento, la propiedad y el control de los medios de comunicación social. En efecto, el fenómeno creciente de comercialización de las comunicaciones ha implicado un debilitamiento del pluralismo informativo como bien público que consagra los derechos a la comunicación. Este es uno de los desafíos principales para el fortalecimiento del sistema informático nacional que pueden desprenderse de este estudio.

En esta misma dirección, a pesar que la perspectiva de los comunicadores indica que las condiciones del pluralismo informativo corresponden a una situación precaria o limitada debido al poder del dinero frente a las líneas editoriales, la percepción de los entrevistados afirma que existe un pluralismo interno a los medios en los cuales se desempeñan. Esta tendencia es argumentada por los entrevistados respecto de los esfuerzos por proveer información desde diversos puntos de vistas contra las limitaciones impuestas por la concentración de la propiedad y el control de los medios. Con ello, cabe concluir que según la percepción de los comunicadores, el ejercicio del periodismo en Chile se encuentra entre diversidad y concentración, poniendo en tensión un concepto integral del pluralismo en los medios desde un enfoque tanto estructural como interno a los medios.

Estos hallazgos cobran particular interés cuando se desea profundizar en un estudio del pluralismo en el sistema informativo nacional desde la pregunta por los medios digitales. Investigaciones sobre las percepciones de los periodistas respecto del pluralismo informativo en Chile se han realizado con anterioridad pero no han abordado a los medios digitales. Hoy en día la comunicación digital adquiere mayor relevancia al ser una de las principales modalidades para el acceso a la información pública, por lo que el análisis de las dinámicas internas y los límites para la transmisión de información y conocimiento por plataformas digitales es más urgente en el contexto actual. En el caso de los medios digitales, resulta interesante que, a diferencia de otros estudios cualitativos de percepciones centrados en los medios tradicionales que evidencian las restricciones internas a los medios sobre el ejercicio periodístico, los hallazgos consignados en este artículo indican que las percepciones de quienes trabajan en los medios digitales identifican las amenazas al pluralismo en las condiciones estructurales del sistema medial. Este artículo contribuye a completar el panorama respecto de las percepciones de los periodistas sobre el pluralismo informativo al explorar la realidad de los medios digitales. Ahora bien, los resultados no pueden ser considerados como ex- 
trapolables al conjunto de los comunicadores de plataformas digitales, sino valoradas como un análisis exploratorio que lanza algunas luces sobre la cuestión, pero que no busca alumbrar todo el panorama.

\section{Notas}

1. Esta investigación ha sido financiada por el proyecto PLU1300008 “El pluralismo en la agenda política de los medios digitales escritos en las regiones $\mathrm{V}$, VIII y Metropolitana" del Programa de Información Científica de la Comisión Nacional de Investigación Científica y Tecnológica de Chile (Nicolás Del Valle Orellana, Investigador Responsable).

\section{Referencias}

Almiron, N. (2006). Pluralismo en Internet: el caso de los diarios digitales españoles de información general sin referente impreso. Ámbitos (15), 9-31.

Arriagada, A., Correa, T., Scherman, A. \& Abarzúa, J. (2015). Santiago no es Chile: brechas, prácticas y percepciones de la representación medial en las audiencias chilenas. Cuadernos. Info (37), 63-75. http://dx.doi.org/10.7764/cdi.37.769

Becerra, M. \& Mastrini, G. (2017). La concentración infocomunicacional en América Latina. Bernal: Universidad Nacional de Quilmes.

Boussaguet, L. (2016). Participatory mechanisms as symbolic policy instruments? Comparative European Politics, 14(1), 107-124. doi:10.1057/cep.2015.12

Breull, L. (2015). Debate: Concentración de medios en la industria televisiva chilena. Recuperado de: https://www.cntv.cl/debate-concentracion-de-medios-en-laindustria-televisiva-chilena/cntv/2016-11-29/122746.html

Campos-Freire, F., Rúas-Araújo, J., López-García, X., \& Martínez-Fernández, V.-A. (2016). El impacto de las redes sociales en el periodismo. El Profesional de la Información, 25(3), 449. https://doi.org/10.3145/epi.2016.may.15

Castells, M. (2001). La Galaxia Internet. Reflexiones sobre Internet, empresa y sociedad. Madrid: Areté.

Castells, M. (2009). Communication Power. Oxford: Oxford University Press.

Castells, M. (2015). "Enredados para la libertad. Movimientos sociales en la era de internet". En Sandoval, R. (Ed.), Pensar desde la resistencia anticapitalista y la autonomía (pp. 51-82), Ciudad de México: CIESAS.

Clemens, E. S. (2010). Democratization and Discourse: The Public Sphere and Comparative Historical Research. Social Science History, 34(3), 373-381. Recuperado de: http://www.jstor.org/stable/4092761 
Coddington, M. (2012). Building frames link by link: the linking practices of blogs and news sites (Report). International journal of communication (Online), 2007. Recuperado de: http://ijoc.org/index.php/ijoc/article/view/1476

Correa, T., Scherman, A., \& Arriagada, A. (2016). Audiences and Disasters: Analyses of Media Diaries Before and After an Earthquake and a Massive Fire. Journal of Communication, 66(4), 519-541. https://doi.org/10.1111/jcom.12245

Czepek, A. (2009). "Pluralism and participation as desired results of press freedom: Measuring media system performance". En Czepek, A., Helwig, M \& Nowak, E. (eds.), Press freedom and pluralism in Europe concepts and conditions (pp. 37-44), Chicago, Estados Unidos: University of Chicago Press. Url: http://press.uchicago.edu/ucp/books/book/distributed/P/bo6921754.html

Dahl, R. (1991). Los Dilemas del Pluralismo Democrático. Autonomía versus control. México D.F.: Alianza Editorial.

Del Valle, N. (2016a). Pluralismo informativo de los medios digitales escritos en Chile: apuntes de investigación. Serie Política, Gestión y Políticas Públicas (3), 1-29. Recuperado de: http://policypapers.cl/2016/pluralismo-informativo-de-los-medios-digitales-escritos-en-chile/

Del Valle, N. (2016b). Pluralismo informativo y libertad en los medios en Chile: notas sobre las condiciones estructurales. Revista de Gestión Pública, V(2), 219-252. http://revistadegestionpublica.cl/index.php/rgp/article/view/73

Domingo, D. \& Heinonen, A. (2008). Weblogs and Journalism. A Typology to Explore the Blurring Boundaries. Nordicom Review, 29(1), 3-15. https://doi.org/10.1515/nor2017-0159

Domingues-da-Silva, J., Zaverucha, J., Figueireido, D. \& Carvalho da Rocha, D. (2015). More concentration of Media ownership means less democracy? Testing association between variables. Intercom - RBCC, 38(1), 65-83. doi: https://doi. org/10.1590/1809-5844201513

Dreyfus, S., Lederman, R., Bosua, R. \& Milton, S. (2011). Can we handle the truth? Whistleblowing to the media in the digital era. Global Media Journal, 5(1), 1, 157-165 Recuperado de: https://acastilloreporter.files.wordpress.com/2014/01/wikileaks-journalism-and-the-21st-century-mediascape1.pdf

Duarte, J., Huertas, M., Rosa, R., \& Caffarena, V. (2015). El papel de las tecnologías cívicas en la redefinición de la esfera pública/The role of civic technologies in the redefinition of the public sphere. Historia y Comunicación Social, 20(2), 483-498. https://doi.org/10.5209/rev_HICS.2015.v20.n2.51396

Fenton, N. (2010). New media, old news: journalism \& democracy in the digital age: London: SAGE. https://us.sagepub.com/en-us/nam/new-media-old-news/ book233055\#description

Fortunati, L., \& Sarrica, M. (2011). Insights from journalists on the future of the press. Communications, 36(2). https://doi.org/10.1515/comm.2011.007

Hansen, E. (2013). Aporias of digital journalism. Journalism, 14(5), 678-694. https://doi. org/10.1177/1464884912453283 
Garnham, N. (1992). "The Media and The Public Sphere". En: C. Calhoun (Ed.), Habermas and the Public Sphere (pp. 357-376). Cambridge: MIT Press.

Garnham, N. (1999). "Amartya Sen's Capabilities Approach to the Evaluation of Welfare: Its Application to Communications". En: A. J.-C. B. Calabrese (Ed.), Communication, Citizenship, and Social Policy. Lanham: Rowman \& Littlefield.

Godoy, S. \& Gronemeyer, M. A. (2012). Los medios digitales: Chile. Open Society Foundations. Recuperado de: https://www.opensocietyfoundations.org/ uploads/3ae7ed67-f633-4a81-bd75-7aa1e784bcdb/mapping-digital-mediachile-sp-20140109_1.pdf

Grazian, D. (2005). A Digital Revolution? A Reassessment of New Media and Cultural Production in the Digital Age. Annals of the American Academy of Political and Social Science, 597, 209-223. https://doi.org/10.1177/0002716204270286

Habermas, J. \& Rawls, J. (1998). Debate Sobre El Liberalismo Político. Barcelona: Paidós.

Hatcher, J. A., \& Currin-Percival, M. (2016). Does the Structural Pluralism Model Predict Differences in Journalists' Perceptions of Online Comments? Digital Journalism, 4(3), 302-320. https://doi.org/10.1080/21670811.2015.1049636

Honneth, A. (2014). Freedom's Right. The Social Foundations of Democratic Life. Cambridge: Polity Press.

International Data Corporation (IDC) (2009). Barómetro Cisco de Banda Ancha Chile 20032010. Trabajo preparado para Cisco. Recuperado el 20 de mayo de 2016, de http://www.subtel.gob.cl/

International Data Corporation (IDC) (2012). Barómetro Cisco de Banda Ancha 2.0. Trabajo preparado para Cisco. Recuperado el 20 de mayo de 2016, de http://www.acti.cl/

Kahne, J., \& Middaugh, E. (2012). Digital Media Shapes Youth Participation in Politics. Phi Delta Kappan, 94(3), 52-56. https://doi.org/10.1177/003172171209400312

Karppinen, K. (2013). Rethinking Media Pluralism. New York: Fordham University Press.

Klinenberg, E. (2005). Convergence: News Production in a Digital Age. The Annals of the American Academy of Political and Social Science, 597, 48-64. https://doi. org/10.1177/0002716204270346

Lloyd, J. (2015). Journalism and PR: new media and public relations in the digital age. London: I.B. Tauris \& Co.

Martinez Arias, S. (2015). Periodismo ciudadano, en los límites de la profesión periodística. Estudios sobre el Mensaje Periodístico, 21 SE, 109. https://doi.org/10.5209/ rev_ESMP.2015.v21.51132

McNeal, R., Mossberger, K. \& Tolbert, C. (Ed.) (2007). Digital Citizenship. The Internet, Society, and Participation. London: MIT Press. http://dx.doi. org/10.1080/19331680802290972

Nielsen, R. P. (1984). Pluralism in the mass media: Can management participation help? Journal of Business Ethics, 3(4), 335-341. https://doi.org/10.1007/BF00381758 
Rawls, J. (1993). Political Liberalism. New York: Columbia University Press.

Rawls, J. (1997). The Idea of Public Reason Revisited. The University of Chicago Law Review, 64(3), 765-807.

Sánchez Gonzales, H. M., \& Méndez Muros, S. (2015). Las guías de uso de medios sociales: regularización periodística y calidad informativa. Estudios sobre el Mensaje Periodístico, 21 SE, 143. doi: https://doi.org/10.5209/rev_ESMP.2015.v21.51136

Sapiezynska, E., Lagos, C. \& Cabalín, C. (2013). Libertad de prensa bajo presión: niveles de restricción percibidos por periodistas chilenos y factores influyentes. Cuadernos.info, (32), 11-26. doi: https://doi.org/10.7764/cdi.32.468

Sartori, G. (2001). La Sociedad Multiétnica. Pluralismo, Multiculturalismo y Extranjeros. Madrid: Taurus.

Scott, B. (2005). A Contemporary History of Digital Journalism. Television \& New Media, 6(1), 89-126. https://doi.org/10.1177/1527476403255824

Stühmeier, T. (2016). Media market concentration and pluralism. CAWM Discussion Paper. Recuperado de: https://www.econstor.eu/handle/10419/146110

Varas-Alarcón, M., \& González Arias, C. (2016). Interactividad en sitios web de medios: buscando nuevas formas de diálogo con sus usuarios. Estudios sobre el Mensaje Periodístico, 22(1), 566. https://doi.org/10.5209/rev_ESMP.2016.v22.n1.52613

Wall, M. (2005). 'Blogs of war': Weblogs as news. Journalism, 6(2), 153-172. https://doi. org/10.1177/1464884905051006

Wall, M. (2015). Citizen Journalism: A retrospective on what we know, an agenda for what we don't. Digital Journalism, 3(6), 797-813. doi: https://doi.org/10.1080/2167081 1.2014. 1002513

Welbers, K., van Atteveldt, W., Kleinnijenhuis, J., Ruigrok, N., \& Schaper, J. (2016). News selection criteria in the digital age: Professional norms versus online audience metrics. Journalism, 17(8), 1037-1053. https://doi.org/10.1177/1464884915595474

Zárate, S. (2016). Pluralismo en el sistema informativo. Temas de la Agenda Pública, 11(88), 1-14. Recuperado de: http://politicaspublicas.uc.cl/wp-content/ uploads/2016/08/PDF-Temas-de-la-Agenda-88.pdf

Zeng, M. A., Dennstedt, B., \& Koller, H. (2016). Democratizing Journalism - How User $\square$ Generated Content and User Communities Affect Publishers' Business Models. Creativity and Innovation Management, 25(4), 536-551. https://doi.org/10.1111/ caim. 12199 
- Sobre los autores:

Nicolás del Valle Orellana es Politólogo y Doctor en Filosofía de la Universidad de Leiden (Países Bajos). Investigador del International Institute for Philosophy and Social Studies, Santiago, Chile. Profesor adjunto en la Escuela de Periodismo de la Universidad de Santiago de Chile.

Fernando Carreño Donoso es Politólogo y Magíster en Comunicación Política de la Universidad de Chile. Investigador del International Institute for Philosophy and Social Studies, Santiago, Chile.

\section{- ¿Cómo citar?}

Del Valle, N. \& Carreño, F. (2020).

Diversos pero concentrados: percepciones de los comunicadores sobre el pluralismo de los medios digitales en Chile. Comunicación y Medios, (42), 30-43. https://doi. org/10.5354/0719-1529.2020.57636 